\title{
State-Dependent Optical Lattices for the Strontium Optical Qubit
}

\author{
A. Heinz,${ }^{1,2, \dagger}$ A. J. Park, ${ }^{1,2, \dagger}$ N. Šantić, ${ }^{1,2}$ J. Trautmann $\odot,{ }^{1,2}$ S. G. Porsev ${ }^{3,4}$ M. S. Safronova ${ }^{3,5}$ \\ I. Bloch, ${ }^{1,2,6}$ and S. Blatt $\oplus^{1,2, *}$ \\ ${ }^{1}$ Max-Planck-Institut für Quantenoptik, Hans-Kopfermann-Straße 1, 85748 Garching, Germany \\ ${ }^{2}$ Munich Center for Quantum Science and Technology, 80799 München, Germany \\ ${ }^{3}$ Department of Physics and Astronomy, University of Delaware, Newark, Delaware 19716, USA \\ ${ }^{4}$ Petersburg Nuclear Physics Institute of NRC "Kurchatov Institute," Gatchina, Leningrad District 188300, Russia \\ ${ }^{5}$ Joint Quantum Institute, National Institute of Standards and Technology and the University of Maryland, \\ College Park, Maryland 20742, USA \\ ${ }^{6}$ Fakultät für Physik, Ludwig-Maximilians-Universität München, 80799 München, Germany
}

(Received 22 December 2019; accepted 20 April 2020; published 18 May 2020)

\begin{abstract}
We demonstrate state-dependent optical lattices for the Sr optical qubit at the tune-out wavelength for its ground state. We tightly trap excited state atoms while suppressing the effect of the lattice on ground state atoms by more than 4 orders of magnitude. This highly independent control over the qubit states removes inelastic excited state collisions as the main obstacle for quantum simulation and computation schemes based on the Sr optical qubit. Our results also reveal large discrepancies in the atomic data used to calibrate the largest systematic effect of Sr optical lattice clocks.
\end{abstract}

DOI: 10.1103/PhysRevLett.124.203201

The experimental implementation of innovative quantum simulation and quantum computation schemes based on the optical qubit in strontium [1-7] has been hindered by the presence of strong inelastic collisions between atoms in the excited qubit state [8]. Although these losses can be suppressed in deep three-dimensional optical lattices [9], such strong trapping precludes using tunneling and elastic collisions [10] to entangle atoms in different lattice sites. Controlled collisional phase gates [7] in particular require high-fidelity, independent control over atoms in either qubit state ${ }^{1} \mathrm{~S}_{0}(g)$ and ${ }^{3} \mathrm{P}_{0}(e)$, shown in Fig. 1(a). Here, we provide a solution to these problems by demonstrating optical lattices at the so-called tune-out wavelength for the ground state $[11,12]$, where its dipole polarizability vanishes, as shown in Fig. 1(b). At this tune-out wavelength, an $e$ atom is tightly trapped, while a $g$ atom is free to move. This condition shuts off the inelastic $e-e$ collisions [8], while allowing the use of the elastic $e-g$ and $g-g$ collisions [10] to engineer novel systems for quantum simulation [1316] and computation [7].

With a novel method, we measure an absolute frequency of $434972130(10) \mathrm{MHz}$ for the tune-out wavelength in ${ }^{88} \mathrm{Sr}$. At the tune-out wavelength, the differential ac Stark shift on the optical qubit transition is only due to the

Published by the American Physical Society under the terms of the Creative Commons Attribution 4.0 International license. Further distribution of this work must maintain attribution to the author(s) and the published article's title, journal citation, and DOI. Open access publication funded by the Max Planck Society. polarizability of the $e$ state. We directly measure this polarizability with Stark shift spectroscopy, demonstrate trapping of $e$ atoms in an optical lattice at the tune-out wavelength, and show that losses from light scattering are small. Given a moderate laser frequency stability corresponding to our measurement uncertainty, $e$ atoms are tightly trapped while the trap's effect on $g$ atoms is suppressed by more than 4 orders of magnitude, the highest level of suppression in any system to date [17-25].
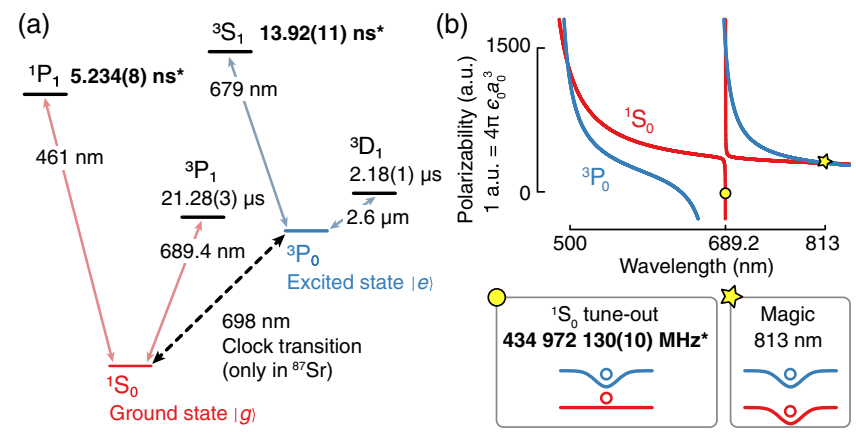

FIG. 1. (a) Simplified Sr level diagram. Optical dipole traps in the red and infrared for the ground $(g)$ and excited $(e)$ qubit (clock) states of Sr are given by coupling to two low-lying singlet and triplet states, respectively. (b) The trap depth for each clock state as a function of wavelength is proportional to the dynamic dipole polarizability. At the "magic wavelength" (star), $g$ and $e$ experience the same trap depth. At the "tune-out wavelength" (circle), an atom in $g$ is free to move, while an atom in $e$ remains trapped. All quantities marked with an asterisk are measured in this Letter. 


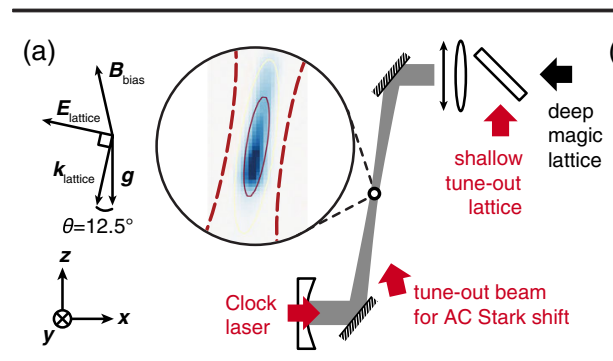

(d)

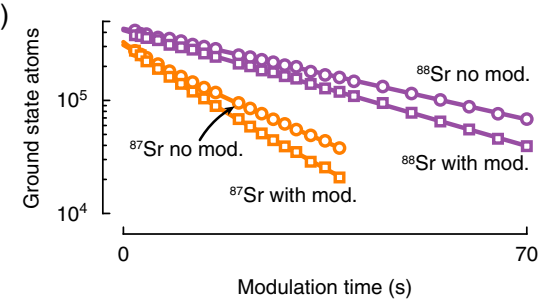

(b)

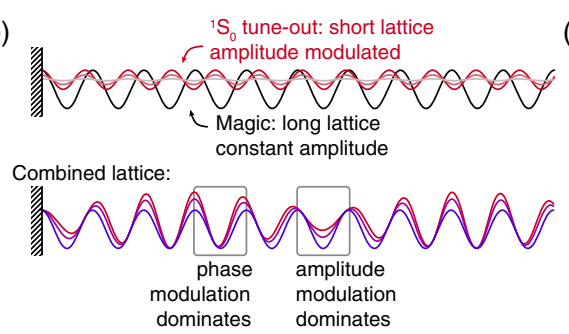

(e)

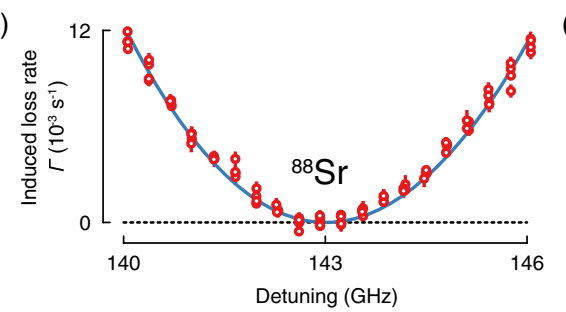

(c)

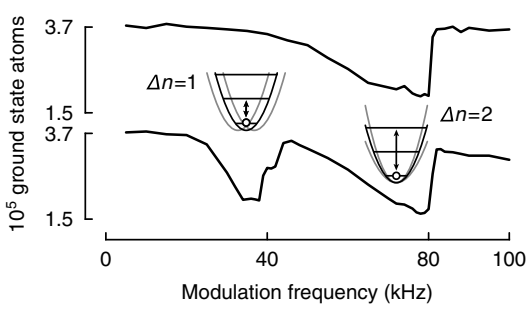

(f)

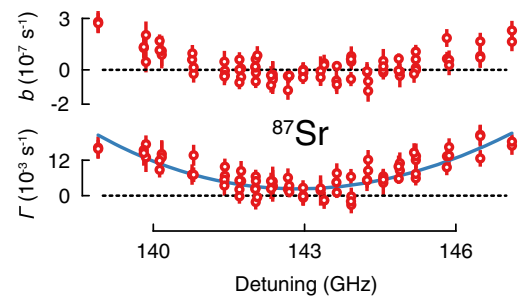

FIG. 2. (a) Experimental setup and optical lattice geometry. We overlap the shallow lattice with the deep magic-wavelength lattice and trap atoms in this combined trap. (b) When modulating the amplitude of the shallow lattice, the atoms are heated due to phase and amplitude modulation of the wells of the optical lattice. (c) Lattice modulation spectrum when modulating the magic-wavelength lattice without the shallow lattice (top) and when modulating the shallow lattice (bottom) in the combined trap. (d) Lifetime measurements for bosonic ${ }^{88} \mathrm{Sr}$ and fermionic ${ }^{87} \mathrm{Sr}$, when the shallow lattice is modulated or not. (e) The induced exponential loss rate $\Gamma$ has a minimum at the detuning from the ${ }^{1} \mathrm{~S}_{0}-{ }^{3} \mathrm{P}_{1}$ transition for ${ }^{88} \mathrm{Sr}$ corresponding to the tune-out wavelength. (f) For ${ }^{87} \mathrm{Sr}$, interactions lead to an induced two-body loss rate $b$ and an increased uncertainty.

We combine these measurements with high-precision atomic structure theory and direct lifetime measurements of the ${ }^{3} \mathrm{P}_{1}$ state [26] to extract new values for the ${ }^{1} \mathrm{P}_{1}$ lifetime. We find a $7 \sigma$ discrepancy to the currently accepted ${ }^{1} \mathrm{P}_{1}$ lifetime from photoassociative spectroscopy [27]. Our polarizability measurements also improve the ${ }^{3} \mathrm{~S}_{1}$ lifetime by an order of magnitude and resolve discrepancies between prior measurements [28-30]. Our results highlight the importance of direct and precise atomic lifetime measurements to bring the accuracy of optical lattice clocks [31] into the $10^{-19}$ regime.

Measuring the tune-out wavelength.-We measure the tune-out wavelength by trapping $g$ atoms in a magicwavelength optical lattice, overlapping an additional optical lattice beam close to the tune-out wavelength and modulating its intensity. The modulation causes parametric heating and trap loss. Minimizing the induced loss allows us to precisely determine the tune-out wavelength. Most measurements of tune-out wavelengths use atom interferometers based on thermal atoms [17-20] or quantumdegenerate gases [21-24]. In contrast, our method is similar to Ref. [25] in that we measure trap loss with an ac method, but with long integration times, reduced systematic effects, and applicability to atoms in excited states, molecules, and trapped ions.

We begin by loading $2 \times 10^{5} \mathrm{~g}$ atoms [32] into a deep near-vertical one-dimensional optical lattice, created by retroreflecting $290 \mathrm{~mW}$ of magic-wavelength laser light at 813.4274(2) nm, as sketched in Fig. 2(a). The magicwavelength lattice has a longitudinal (transverse) trap frequency of $\sim 40 \mathrm{kHz}(\sim 100 \mathrm{~Hz})$, corresponding to a lattice depth of $k_{B} \times 5.6 \mu \mathrm{K}=h \times 116 \mathrm{kHz}$ and a $1 / e^{2}$ beam waist of $75 \mu \mathrm{m}$, where $k_{B}(h=2 \pi \hbar)$ is the Boltzmann (Planck) constant. The atoms occupy an ellipsoid with a $1 / e^{2}$ diameter of $\sim 240 \mu \mathrm{m}(\sim 80 \mu \mathrm{m})$ along the longitudinal (transverse) trap axis, with a typical lattice site filling 500, as shown in Fig. 2(a). From such in situ and time-of-flight absorption images, we determine atom numbers and temperatures [32].

We then overlap the deep magic-wavelength lattice with a shallow one-dimensional lattice created by retroreflecting $4.5 \mathrm{~mW}$ of light near the tune-out wavelength, as shown in Fig. 2(a). This geometry allows us to heat the atoms in the combined lattice by intensity modulating the shallow lattice, as sketched in Fig. 2(b). Since the two lattices are incommensurate there will be lattice sites in which heating due to phase modulation dominates, while in others heating due to amplitude modulation dominates. By changing the modulation frequency and observing atom loss from the trap [33], we obtain spectra as shown in Fig. 2(c). We take a reference spectrum (top) by modulating the deep lattice intensity, while the shallow lattice is turned off. In this case, we observe a single minimum in the spectrum at $f_{\text {mod }} \simeq 80 \mathrm{kHz}$, corresponding to amplitude modulation and parametric heating [33] that results in transitions between lattice bands that are two motional quanta apart. The response of the combined lattice due to intensity modulation of the shallow lattice (bottom) shows another minimum at $\sim 40 \mathrm{kHz}$, corresponding to phase modulation and transitions between adjacent lattice bands [33].

To compare the effect of heating at different wavelengths of the shallow lattice, we intensity modulate it at $f_{\text {mod }}$ and 
measure the resulting exponential trap loss rate. Other loss mechanisms such as losses due to intensity noise of both lattices, collisions with background gas atoms, and photon scattering losses, also contribute to the measured heating rate. We determine the induced trap loss rate $\Gamma(\omega)$ by taking the difference between the measured loss rate without modulation and with modulation. Examples of such measurements are shown in Fig. 2(d) for ${ }^{88} \mathrm{Sr}$ (top) and ${ }^{87} \mathrm{Sr}$ (bottom). The ${ }^{88} \mathrm{Sr}$ data are well described by an exponential decay because of the isotope's vanishingly small scattering length. In contrast, the ${ }^{87} \mathrm{Sr}$ data show additional superexponential two-body decay. This decay is due to elastic interactions $[34,35]$ that lead to evaporative trap loss, which we fit with a two-body decay term [36]. The induced trap loss rate vanishes when the ground state polarizability $\alpha_{g}$ crosses zero at the tune-out wavelength, and it is proportional to [36]

$$
\Gamma(\omega) \propto \alpha_{g}^{2}(\omega) I_{\text {mod }}^{2} f_{\text {mod }}^{-2},
$$

where $\omega$ is the optical frequency of the tune-out laser, and $I_{\text {mod }}$ is the intensity modulation depth. To compensate for drifts in $I_{\text {mod }}$ and the trap frequency, we normalize the measured $\Gamma(\omega)$ according to Eq. (1). The wavelength of the shallow lattice laser is locked to a wave meter but measured with a self-referenced femtosecond frequency comb, resulting in an absolute frequency error of $3 \mathrm{MHz}$.

The normalized data for ${ }^{88} \mathrm{Sr}$ and ${ }^{87} \mathrm{Sr}$ are shown in Figs. 2(e) and 2(f), respectively, as a function of detuning from each isotope's ${ }^{1} \mathrm{~S}_{0}-{ }^{3} \mathrm{P}_{1}$ transition [47]. The induced loss rate $\Gamma$ for both isotopes shows a minimum at detuning $\Delta_{t}$, corresponding to the tune-out wavelength for each isotope. For ${ }^{87} \mathrm{Sr}$, the induced two-body loss coefficient $b$, given by the difference of the two-body coefficients extracted from the underlying atomic decay curves, shows the same behavior with respect to detuning as $\Gamma$. This behavior can be explained by an increased tunneling rate in the second lattice band, leading to increased evaporation, correlated exponential and two-body decay rates, and an increased uncertainty for $\Delta_{t}^{87}$. We model the induced loss rate as $\Gamma(\Delta)=c_{0}\left(1-\Delta_{t} / \Delta\right)^{2}[36]$, where $\Delta \equiv \omega-\omega_{3 \mathrm{P}_{1}}$ is the detuning from the isotope-shifted ${ }^{1} \mathrm{~S}_{0}-{ }^{3} \mathrm{P}_{1}$ transition, and the unused fit parameter $c_{0}$ relates the parametric heating rate to the trap loss rate [33]. We find $\Delta_{t}^{88}=$ $2 \pi \times 143.009(8) \mathrm{GHz}$ and $\Delta_{t}^{87}=2 \pi \times 142.86(8) \mathrm{GHz}$ for ${ }^{88} \mathrm{Sr}$ and ${ }^{87} \mathrm{Sr}$, respectively. These numbers are in good agreement, considering the empirical two-body loss model for ${ }^{87} \mathrm{Sr}$. In the Supplemental Material [36], we derive a conservative upper limit $\left|\Delta_{t}^{88}-\Delta_{t}^{87}\right|<2 \pi \times 23 \mathrm{MHz}$ due to hyperfine splitting, vector, and tensor shifts. In the following, we use the measured $\Delta_{t}^{88}$ for ${ }^{87} \mathrm{Sr}$ and suppress the superscript for clarity. This choice leads to a residual $\alpha_{g}= \pm 0.05$ a.u. from the 2.4 a.u. $/ \mathrm{GHz}$ polarizability slope around $\Delta_{t}$. Here 1 a.u. $=4 \pi \epsilon_{0} a_{0}^{3}$ is the atomic unit of polarizability, and $\epsilon_{0}\left(a_{0}\right)$ is the vacuum permittivity (Bohr radius).

To minimize systematic shifts in $\Delta_{t}$ due to laser noise, unsuppressed longitudinal laser modes, and amplified spontaneous emission, we Fourier filter [36] the shallow lattice laser and suppress light at the ${ }^{1} \mathrm{~S}_{0}-{ }^{3} \mathrm{P}_{1}$ transition by $>90 \mathrm{~dB}$ compared to the carrier. To avoid saturation of $\Gamma$, and to work with the same $I_{\text {mod }}$ throughout, we limit the measurement range to a few gigahertz. Reducing the measurement range further does not change $\Delta_{t}$ significantly, and we estimate saturation effects to be negligible.

For ${ }^{87} \mathrm{Sr}$, we observe and fit a statistically significant offset of $2.3(4) \times 10^{-3} \mathrm{~s}^{-1}$ in the induced trap loss rate. While the offset for ${ }^{87} \mathrm{Sr}$ could be explained by contributions from vector and tensor polarizabilities, these cannot occur in ${ }^{88} \mathrm{Sr}$. When fitting the ${ }^{88} \mathrm{Sr}$ data with an offset, we find a much smaller value of $2(1) \times 10^{-4} \mathrm{~s}^{-1}$, which also causes a systematic shift of the tune-out frequency of $2 \mathrm{MHz}$. In conclusion, we find that the tune-out frequency $\omega_{t}$ for the ground state is detuned from the ${ }^{1} \mathrm{~S}_{0}-{ }^{3} \mathrm{P}_{1}$ transition at $\omega_{3 \mathrm{P}_{1}}$ by

$$
\Delta_{t} / 2 \pi=(143.009 \mathrm{GHz}) \pm(8 \mathrm{MHz})_{\text {stat }} \pm(2 \mathrm{MHz})_{\mathrm{sys}} .
$$

Measuring the excited state polarizability.-Since the polarizability of the $g$ state vanishes at $\omega_{t}$, the ac Stark shift on the clock transition induced by a laser beam at $\omega_{t}$ is solely determined by the excited state polarizability. For this reason, we can directly measure the $e$ state polarizability $\alpha_{e}\left(\omega_{t}\right)$ by Stark shift spectroscopy. For these measurements, we prepare a sample of ${ }^{87} \mathrm{Sr}$ in a onedimensional magic-wavelength lattice. We propagate a clock laser beam at $3.5^{\circ}$ with respect to the lattice axis and overlap it with the lattice at the position of the atoms. A typical spectrum of the clock transition is shown in Fig. 3(a), consistent with a $\sim 1 \mu \mathrm{K}$ temperature [48], confirmed by time-of-flight data. On the carrier transition, we observe damped Rabi oscillations, consistent with the mismatch between the lattice axis and the clock laser wave vector [48]. For the following measurements, we illuminate the atoms with the clock laser for $0.3 \mathrm{~ms}$, corresponding to the maximum excited state fraction. For the ac Stark shift spectroscopy, we additionally apply a laser beam at $\omega_{t}$ with a $1 / e^{2}$ waist of $300 \mu \mathrm{m}$, as illustrated in Fig. 2(a). As a function of clock laser detuning, we observe the spectra shown in Fig. 3(b) and fit them to extract the center frequencies. To calibrate the intensity, we load a sample of ${ }^{88} \mathrm{Sr}$ into the same magic-wavelength lattice at the same position as the ${ }^{87} \mathrm{Sr}$ sample. After diabatically switching off the lattice, we measure free-space Rabi oscillations on each of the three ${ }^{1} \mathrm{~S}_{0}-{ }^{3} \mathrm{P}_{1}$ transitions in a small magnetic bias field, as shown in Fig. 3(c). We fit the Rabi oscillations with an analytic solution to the optical Bloch equations [49] and 

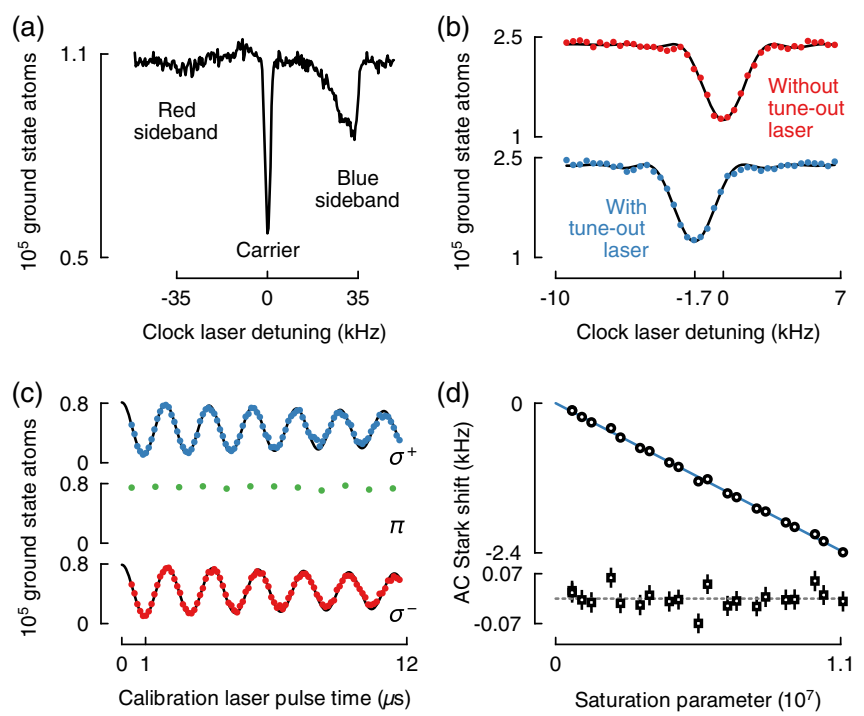

FIG. 3. (a) Sideband spectrum of the clock transition in the magic-wavelength lattice. (b) Typical ac Stark shift spectra obtained on the carrier transition. (c) Resonant free-space Rabi oscillations for ${ }^{88} \mathrm{Sr}$ calibrate the polarization and intensity of the Stark-shifting beam. (d) The Rabi frequencies calibrate the saturation parameter for the measured ac Stark shift (circles). We extract the $e$ state polarizability from the slope of the $\chi_{\text {red }}^{2}=$ 0.94 linear fit (solid line). Fit residuals are shown as squares.

extract the Rabi frequency $\Omega_{ \pm}$for each $\sigma^{ \pm}$polarization component, for an applied power $P_{0}=112 \mu \mathrm{W}$. The Rabi frequency of the Stark-shifting beam with power $P$ is calibrated as $\Omega^{2} \equiv\left(\Omega_{+}^{2}+\Omega_{-}^{2}\right) P / P_{0}$, which allows expressing the ac Stark shift of the clock transition at the tune-out frequency $\Delta \omega_{e g}=-\omega_{3 \mathrm{P}_{1}}^{3} \tau_{3 \mathrm{P}_{1}} \alpha_{e}\left(\omega_{t}\right) \Omega^{2} /$ $\left(12 \pi \epsilon_{0} c^{3}\right)$ in terms of measured quantities, where $c$ is the speed of light. In Figure 3(d), we plot $\Delta \omega_{e g}$ as a function of the saturation parameter $s_{0}=2 \Omega^{2} \tau_{3 \mathrm{P}_{1}}^{2}$ and use a linear fit to extract the excited state polarizability

$$
\alpha_{e}\left(\omega_{t}\right)=\left(1555 \pm 8_{\text {stat }} \pm 2_{\text {sys }}\right) \text { a.u. }
$$

of ${ }^{87} \mathrm{Sr}$ at the tune-out frequency, where the systematic uncertainty includes the mismatch between the tune-out frequency for ${ }^{88} \mathrm{Sr}$ and ${ }^{87} \mathrm{Sr}$, but is dominated by the effect of the laser spectrum. Our measured polarizability agrees well with our theoretical prediction of 1546(14) a.u., based on Ref. [50].

Trapping excited atoms at the tune-out wavelength.We prepare a sample of $e$ state ${ }^{87} \mathrm{Sr}$ atoms in the magicwavelength lattice and transfer them to the tune-out lattice [36]. In Fig. 4, we compare the number of $e$ atoms in the tune-out lattice as a function of hold time to the case where we trap $e$ atoms in the magic-wavelength lattice. In both cases, the atoms decay superexponentially via $e$-e collisions [8]. In the magic-wavelength lattice, this inelastic loss dominates, while the atoms in the tune-out lattice

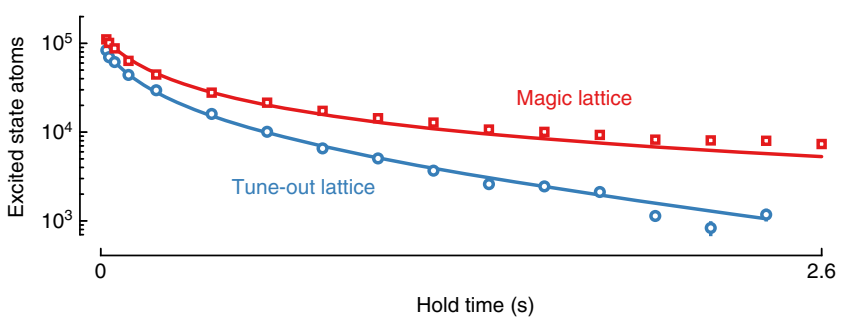

FIG. 4. Number of $e$ atoms versus hold time in a one-dimensional optical lattice at the magic wavelength (squares) and at the tune-out wavelength (circles). Both lattices exhibit the expected losses due to inelastic collisions, while the tune-out lattice additionally exhibits exponential one-body decay due to light scattering.

experience additional exponential loss with a $1 / e$ lifetime $\sim 1.2 \mathrm{~s}$. The intensity of the tune-out lattice is chosen to match the trap frequencies of the magic-wavelength and tune-out lattices at $\sim 40 \mathrm{kHz}$, corresponding to a lattice depth of $\sim 17 \times \hbar \omega_{\text {rec }}\left(\omega_{\text {rec }} / 2 \pi=4.8 \mathrm{kHz}\right.$ is the lattice recoil frequency), confirmed by parametric heating. The measured lifetime agrees well with the theoretically predicted loss due to photon scattering for each lattice axis of $24 \mathrm{~s}$ per recoil of lattice depth. Depending on the application, a compromise between lattice depth and tunneling rate needs to be found. For instance, a two-dimensional tune-out lattice trapping $e$ atoms in a Mott insulator state would have a lifetime $\sim 1 \mathrm{~s}$.

Determination of atomic lifetimes. - The $g$ state polarizability $\quad \alpha_{g}(\omega)=\alpha_{g}\left({ }^{1} \mathrm{P}_{1} ; \omega\right)+\alpha_{g}\left({ }^{3} \mathrm{P}_{1} ; \omega\right)+\alpha_{v c}(\omega)$ is dominated by coupling to the ${ }^{1} \mathrm{P}_{1}$ and ${ }^{3} \mathrm{P}_{1}$ states. All other contributions from valence and core electronic states are small and we calculate their combined value $\alpha_{v c}$ with percent-level precision [50]. Since both ${ }^{1} \mathrm{P}_{1}$ and ${ }^{3} \mathrm{P}_{1}$ decay only to $g$, and because the tune-out frequency is far detuned with respect to each state's natural linewidth, each contribution $\alpha_{g}(j ; \omega) \propto \tau_{j}^{-1}$ [36], where $\tau_{j}$ is the state's natural lifetime. At the tune-out frequency $\alpha_{g}$ vanishes, leading to a strong constraint on the relationship between the lifetimes $\tau_{\mathrm{P}_{1}}$ and $\tau_{\mathrm{P}_{1}}$. While $\tau_{\mathrm{P}_{1}}$ has been recently measured directly [26], the currently accepted value for $\tau_{\mathrm{P}_{1}}$ comes from photoassociative spectroscopy $[27,51]$. Using our tune-out wavelength and $\tau_{3 \mathrm{P}_{1}}$ [26], we find $\tau_{\mathrm{P}_{\mathrm{P}_{1}}}=5.234(8) \mathrm{ns}$ [36], a $7 \sigma$ discrepancy with the currently accepted value [27]. The $e$ state polarizability $\alpha_{e}$ at the tune-out wavelength is dominated $(87 \%)$ by the ${ }^{3} \mathrm{P}_{0}-{ }^{3} \mathrm{~S}_{1}$ transition. We determined all other contributions with a total uncertainty of 4 a.u. using a high-precision relativistic method [50]. Combining these theoretical values with our measurement of $\alpha_{e}$ determines the ${ }^{3} \mathrm{P}_{0}-{ }^{3} \mathrm{~S}_{1}$ matrix element. The ${ }^{3} \mathrm{~S}_{1}$ state dominantly decays to the ${ }^{3} \mathrm{P}_{J}$ levels, while its decay to ${ }^{1} \mathrm{P}_{1}$ is negligible at the present level of accuracy. We calculated the ${ }^{3} \mathrm{~S}_{1}-{ }^{3} \mathrm{P}_{J}$ branching ratios with $0.1 \%$ accuracy, which allows us to extract $\tau_{3 \mathrm{~S}_{1}}$ from the ${ }^{3} \mathrm{P}_{0}-{ }^{3} \mathrm{~S}_{1}$ matrix element. 
This accurate prediction of branching ratios is possible due to very similar electronic correlation effects for these transitions, which largely cancel for their ratios. We find $\tau_{3 \mathrm{~S}_{1}}=13.92(11) \mathrm{ns}$, an improvement of an order of magnitude over prior measurements that ranged from 10.9(1.1) to $15.0(8)$ ns [28-30]. We note in passing that the currently best values for $\tau_{3 \mathrm{P}_{1}}$ and the lifetime of the ${ }^{3} \mathrm{D}_{1}$ state are correlated because they are extracted from a single dataset [26]. The ${ }^{3} \mathrm{D}_{1}$ lifetime directly determines the dynamic contribution to the Sr lattice clock blackbody radiation shift [50], its currently largest systematic uncertainty [52]. This uncertainty can be directly improved by a new direct measurement of $\tau_{\mathrm{P}_{1}}$ in combination with our results and Ref. [26]. Our measurements show that direct measurements of atomic lifetimes and improvements to atomic structure calculations will be essential in bringing optical frequency standards to the $10^{-19}$ level.

In conclusion, we have demonstrated state-dependent optical lattices for the clock states of strontium at the tuneout wavelength for its ground state. With a new spectroscopic method, we achieved a record suppression of the lattice depth for the ground state of more than 4 orders of magnitude. Our method can be applied to thermal gases of atoms, molecules [53], or to trapped ions. As a modulation technique for trapped particles, the method benefits from suppression of systematic errors and long integration times. Using our technique in three-dimensional optical lattices in combination with band mapping [54] will enable measurements of excited state tune-out wavelengths, such as the tune-out wavelength for the ${ }^{3} \mathrm{P}_{0}$ state around $633 \mathrm{~nm}$ [12], even in the presence of interactions. We have demonstrated high-fidelity, state-dependent control of the strontium optical qubit. Combining our results with single-site addressing and control [55] removes the main obstacle for the realization of quantum computation and quantum simulation schemes with two-electron atoms [7]. Finally, our Letter creates new opportunities to use state-dependent optical lattices for quantum simulations of nanophotonics [13-15] and quantum chemistry [16].

We thank J. Ye, A. González-Tudela, J. I. Cirac, and M. J. Martin for stimulating discussions. This work was supported by funding from the European Union H2020 (UQUAM Grant No. 319278 and PASQuanS Grant No. 817482). A. J. P. was supported by a fellowship from the Natural Sciences and Engineering Research Council of Canada (NSERC), funding Ref. No. 517029, and N. Š. was supported by a Marie Skłodowska-Curie individual fellowship, Grant Agreement No. 844161. Work at U. Delaware was performed under the sponsorship of the U.S. Department of Commerce, National Institute of Standards and Technology. S. G. P. acknowledges support by the Russian Science Foundation under Grant No. 1912-00157. *sebastian.blatt@mpq.mpg.de

${ }^{\dagger}$ A. H. and A. J. P. contributed equally to this work.

[1] A. J. Daley, M. M. Boyd, J. Ye, and P. Zoller, Quantum Computing with Alkaline-Earth-Metal Atoms, Phys. Rev. Lett. 101, 170504 (2008).

[2] A. V. Gorshkov, A. M. Rey, A. J. Daley, M. M. Boyd, J. Ye, P. Zoller, and M. D. Lukin, Alkaline-Earth-Metal Atoms as Few-Qubit Quantum Registers, Phys. Rev. Lett. 102, 110503 (2009).

[3] A. V. Gorshkov, M. Hermele, V. Gurarie, C. Xu, P. S. Julienne, J. Ye, P. Zoller, E. Demler, M. D. Lukin, and A. M. Rey, Two-orbital SU(N) magnetism with ultracold alkaline-earth atoms, Nat. Phys. 6, 289 (2010).

[4] M. Foss-Feig, M. Hermele, and A. M. Rey, Probing the Kondo lattice model with alkaline-earth-metal atoms, Phys. Rev. A 81, 051603(R) (2010).

[5] M. Foss-Feig, M. Hermele, V. Gurarie, and A. M. Rey, Heavy fermions in an optical lattice, Phys. Rev. A 82, 053624 (2010).

[6] A. J. Daley, J. Ye, and P. Zoller, State-dependent lattices for quantum computing with alkaline-earth-metal atoms, Eur. Phys. J. D 65, 207 (2011).

[7] A. J. Daley, Quantum computing and quantum simulation with group-II atoms, Quantum Inf. Process. 10, 865 (2011).

[8] M. Bishof, M. J. Martin, M. D. Swallows, C. Benko, Y. Lin, G. Quéméner, A. M. Rey, and J. Ye, Inelastic collisions and density-dependent excitation suppression in a ${ }^{87} \mathrm{Sr}$ optical lattice clock, Phys. Rev. A 84, 052716 (2011).

[9] S. L. Campbell, R. B. Hutson, G. E. Marti, A. Goban, N. D. Oppong, R. L. McNally, L. Sonderhouse, J. M. Robinson, W. Zhang, B. J. Bloom, and J. Ye, A Fermi-degenerate three-dimensional optical lattice clock, Science 358, 90 (2017).

[10] M. J. Martin, M. Bishof, M. D. Swallows, X. Zhang, C. Benko, J. von Stecher, A. V. Gorshkov, A. M. Rey, and J. Ye, A quantum many-body spin system in an optical lattice clock, Science 341, 632 (2013).

[11] Y. Cheng, J. Jiang, and J. Mitroy, Tune-out wavelengths for the alkaline-earth-metal atoms, Phys. Rev. A 88, 022511 (2013).

[12] M. S. Safronova, Z. Zuhrianda, U. I. Safronova, and C. W. Clark, Extracting transition rates from zero-polarizability spectroscopy, Phys. Rev. A 92, 040501(R) (2015).

[13] I. de Vega, D. Porras, and J. I. Cirac, Matter-Wave Emission in Optical Lattices: Single Particle and Collective Effects, Phys. Rev. Lett. 101, 260404 (2008).

[14] L. Krinner, M. Stewart, A. Pazmiño, J. Kwon, and D. Schneble, Spontaneous emission of matter waves from a tunable open quantum system, Nature (London) 559, 589 (2018).

[15] A. González-Tudela and J. I. Cirac, Non-Markovian quantum optics with three-dimensional state-dependent optical lattices, Quantum 2, 97 (2018).

[16] J. Argüello-Luengo, A. González-Tudela, T. Shi, P. Zoller, and J. I. Cirac, Analogue quantum chemistry simulation, Nature (London) 574, 215 (2019).

[17] G. Lamporesi, J. Catani, G. Barontini, Y. Nishida, M. Inguscio, and F. Minardi, Scattering in Mixed Dimensions with Ultracold Gases, Phys. Rev. Lett. 104, 153202 (2010). 
[18] W. F. Holmgren, R. Trubko, I. Hromada, and A. D. Cronin, Measurement of a Wavelength of Light for Which the Energy Shift for an Atom Vanishes, Phys. Rev. Lett. 109, 243004 (2012).

[19] R. Trubko, M. D. Gregoire, W. F. Holmgren, and A. D. Cronin, Potassium tune-out wavelength measurement using atom interferometry and a multipass optical cavity, Phys. Rev. A 95, 052507 (2017).

[20] E. Copenhaver, K. Cassella, R. Berghaus, and H. Müller, Measurement of a ${ }^{7} \mathrm{Li}$ tune-out wavelength by phasepatterned atom interferometry, Phys. Rev. A 100, 063603 (2019).

[21] C. D. Herold, V. D. Vaidya, X. Li, S. L. Rolston, J. V. Porto, and M. S. Safronova, Precision Measurement of Transition Matrix Elements via Light Shift Cancellation, Phys. Rev. Lett. 109, 243003 (2012).

[22] R. H. Leonard, A. J. Fallon, C. A. Sackett, and M. S. Safronova, High-precision measurements of the ${ }^{87} \mathrm{Rb} D$ line tune-out wavelength, Phys. Rev. A 92, 052501 (2015).

[23] F. Schmidt, D. Mayer, M. Hohmann, T. Lausch, F. Kindermann, and A. Widera, Precision measurement of the ${ }^{87} \mathrm{Rb}$ tune-out wavelength in the hyperfine ground state $F=1$ at 790 nm, Phys. Rev. A 93, 022507 (2016).

[24] W. Kao, Y. Tang, N. Q. Burdick, and B. L. Lev, Anisotropic dependence of tune-out wavelength near dy 741-nm transition, Opt. Express 25, 3411 (2017).

[25] B. M. Henson, R. I. Khakimov, R. G. Dall, K. G. H. Baldwin, L.-Y. Tang, and A. G. Truscott, Precision Measurement for Metastable Helium Atoms of the $413 \mathrm{~nm}$ Tune-Out Wavelength at Which the Atomic Polarizability Vanishes, Phys. Rev. Lett. 115, 043004 (2015).

[26] T. Nicholson, S. Campbell, R. Hutson, G. Marti, B. Bloom, R. McNally, W. Zhang, M. Barrett, M. Safronova, G. Strouse, W. Tew, and J. Ye, Systematic evaluation of an atomic clock at $2 \times 10^{-18}$ total uncertainty, Nat. Commun. 6, 6896 (2015).

[27] M. Yasuda, T. Kishimoto, M. Takamoto, and H. Katori, Photoassociation spectroscopy of ${ }^{88} \mathrm{Sr}$ : Reconstruction of the wave function near the last node, Phys. Rev. A 73, 011403(R) (2006).

[28] U. Brinkmann, Lebensdauern und Oszillatorstärken im Sr I- und Ca I-Spektrum, Z. Phys. 228, 440 (1969).

[29] M. D. Havey, L. C. Balling, and J. J. Wright, Direct measurements of excited-state lifetimes in $\mathrm{Mg}, \mathrm{Ca}$, and $\mathrm{Sr}$, J. Opt. Soc. Am. 67, 488 (1977).

[30] G. Jönsson, C. Levinson, A. Persson, and C.-G. Wahlström, Natural radiative lifetimes in the ${ }^{1} \mathrm{P}_{1}$ and ${ }^{1} \mathrm{~F}_{3}$ sequences of $\mathrm{Sr}$ I, Z. Phys. A 316, 255 (1984).

[31] A. D. Ludlow, M. M. Boyd, J. Ye, E. Peik, and P. O. Schmidt, Optical atomic clocks, Rev. Mod. Phys. 87, 637 (2015).

[32] S. Snigirev, A. J. Park, A. Heinz, I. Bloch, and S. Blatt, Fast and dense magneto-optical traps for strontium, Phys. Rev. A 99, 063421 (2019).

[33] T. A. Savard, K. M. O'Hara, and J. E. Thomas, Laser-noiseinduced heating in far-off resonance optical traps, Phys. Rev. A 56, R1095 (1997).

[34] Y. N. Martinez de Escobar, P. G. Mickelson, P. Pellegrini, S. B. Nagel, A. Traverso, M. Yan, R. Côté, and T. C. Killian,
Two-photon photoassociative spectroscopy of ultracold ${ }^{88}$ Sr, Phys. Rev. A 78, 062708 (2008).

[35] A. Stein, H. Knöckel, and E. Tiemann, The $1 S+1 S$ asymptote of $\mathrm{Sr}_{2}$ studied by Fourier-transform spectroscopy, Eur. Phys. J. D 57, 171 (2010).

[36] See Supplemental Material at http://link.aps.org/ supplemental/10.1103/PhysRevLett.124.203201 for our analysis of parametric heating in incommensurate lattices, details on fitting and rescaling of trap loss, analysis of effects which determine the ${ }^{1} \mathrm{~S}_{0}$ tune-out frequency, details on filtering of amplified spontaneous emission, and the experimental procedure of loading excited state atoms into the optical lattice at the tune-out wavelength, which includes Refs. [37-46].

[37] M. Gehm, K. O'Hara, T. Savard, and J. Thomas, Dynamics of noise-induced heating in atom traps, Phys. Rev. A 58, 3914 (1998).

[38] M. Gehm, K. O’Hara, T. Savard, and J. Thomas, Dynamics of noise-induced heating in atom traps, Phys. Rev. A 58, 3914 (1998); Erratum, Phys. Rev. A 61, 029902 (2000).

[39] N. L. Manakov, V. D. Ovsiannikov, and L. P. Rapoport, Atoms in a laser field, Phys. Rep. 141, 320 (1986).

[40] F. L. Kien, P. Schneeweiss, and A. Rauschenbeutel, Dynamical polarizability of atoms in arbitrary light fields: General theory and application to cesium, Eur. Phys. J. D 67, 92 (2013).

[41] M. M. Boyd, High precision spectroscopy of strontium in an optical lattice: Towards a new standard for frequency and time, Ph.D. thesis, Department of Physics, University of Colorado, 2007.

[42] M. Boyd, T. Zelevinsky, A. Ludlow, S. Blatt, T. ZanonWillette, S. Foreman, and J. Ye, Nuclear spin effects in optical lattice clocks, Phys. Rev. A 76, 022510 (2007).

[43] G. Ferrari, P. Cancio, R. Drullinger, G. Giusfredi, N. Poli, M. Prevedelli, C. Toninelli, and G. M. Tino, Precision Frequency Measurement of Visible Intercombination Lines of Strontium, Phys. Rev. Lett. 91, 243002 (2003).

[44] J. E. Sansonetti and G. Nave, Wavelengths, transition probabilities, and energy levels for the spectrum of neutral strontium (Sr I), J. Phys. Chem. Ref. Data 39, 033103 (2010).

[45] M. G. Kozlov and S. G. Porsev, Polarizabilities and hyperfine structure constants of the low-lying levels of barium, Eur. Phys. J. D 5, 59 (1999).

[46] M. S. Safronova, W. R. Johnson, and A. Derevianko, Relativistic many-body calculations of energy levels, hyperfine constants, electric-dipole matrix elements, and static polarizabilities for alkali-metal atoms, Phys. Rev. A 60, 4476 (1999).

[47] H. Miyake, N. C. Pisenti, P. K. Elgee, A. Sitaram, and G. K. Campbell, Isotope shift spectroscopy of the ${ }^{1} \mathrm{~S}_{0} \rightarrow{ }^{3} \mathrm{P}_{1}$ and ${ }^{1} \mathrm{~S}_{0} \rightarrow{ }^{3} \mathrm{P}_{0}$ transitions in strontium, Phys. Rev. Research $\mathbf{1}$, 033113 (2019).

[48] S. Blatt, J. W. Thomsen, G. K. Campbell, A. D. Ludlow, M. D. Swallows, M. J. Martin, M. M. Boyd, and J. Ye, Rabi spectroscopy and excitation inhomogeneity in a one-dimensional optical lattice clock, Phys. Rev. A 80, 052703 (2009).

[49] R. Loudon, The Quantum Theory of Light, 3rd ed. (Oxford University Press, New York, 2000). 
[50] M. S. Safronova, S. G. Porsev, U. I. Safronova, M. G. Kozlov, and C. W. Clark, Blackbody-radiation shift in the Sr optical atomic clock, Phys. Rev. A 87, 012509 (2013).

[51] S. B. Nagel, P. G. Mickelson, A. D. Saenz, Y. N. Martinez, Y. C. Chen, T. C. Killian, P. Pellegrini, and R. Côté, Photoassociative Spectroscopy at Long Range in Ultracold Strontium, Phys. Rev. Lett. 94, 083004 (2005).

[52] T. Bothwell, D. Kedar, E. Oelker, J. M. Robinson, S. L. Bromley, W. L. Tew, J. Ye, and C. J. Kennedy, JILA SrI optical lattice clock with uncertainty of $2.0 \times 10^{-18}$, Metrologia 56, 065004 (2019).
[53] R. Bause, M. Li, A. Schindewolf, X.-Y. Chen, M. Duda, S. Kotochigova, I. Bloch, and X.-Y. Luo, Tune-out and magic wavelengths for ground-state ${ }^{23} \mathrm{Na}{ }^{40} \mathrm{~K}$ molecules, arXiv:1912.10452v2.

[54] S. Blatt, A. Mazurenko, M. F. Parsons, C. S. Chiu, F. Huber, and M. Greiner, Low-noise optical lattices for ultracold ${ }^{6} \mathrm{Li}$, Phys. Rev. A 92, 021402(R) (2015).

[55] C. Weitenberg, M. Endres, J. F. Sherson, P. Schauß, T. Fukuhara, I. Bloch, and S. Kuhr, Single-spin addressing in an atomic Mott insulator, Nature (London) 471, 319 (2011). 